sie allerdings nicht als so überlegen und kompetent an und fordern zum Beispiel in Auseinandersetzungen mit Jungen nicht ihre Rechte ein. Der Slogan „Girl Power“ geht also nicht automatisch mit dem Selbstwertgefühl und der Handlungsmächtigkeit von realen Mädchen einher. In Selbstporträts der Mädchen, in denen sie schildern, wie sie sich sehen und wie sie gerne aussehen möchten, wird ein Hintergrund hierzu deutlich: Sie nehmen ihren eigenen Körper vor allem als defizitär wahr, haben das Gefühl zu dick und nicht hübsch zu sein. Das Aussehen wird in allen Dimensionen zur zentralen Bewertungskategorie von und für Mädchen. Dabei sind auch die Mädchen selber sehr schnell in der Abwertung bestimmter Fernsehfiguren, die ihrer Ansicht nach nicht normal sind. Dieses Bild von Normalität entspricht dabei dem Aussehen der klassischen Barbie, hellhäutig, sehr schlank und mit langen blonden Haaren. Mit diesem Maßstab für „normal“ im Kopf nehmen sie die Defizite an sich wahr. Girl Power mit seinen expliziten Verweisen auf eine bestimmte Körperlichkeit - sehr dünn und sexy - ist die Grundlage für Selbstbestimmtheit und Kraft. Doch einen solchen Körper haben Mädchen nicht. Dies verschärft sich noch einmal für Mädchen, die keinen europäisch-amerikanischen ethnischen Hintergrund haben.

In ihrer Analyse lässt Rebecca Hains die Mädchen pointiert zu Wort kommen. Sie zeichnet ihr methodisches Herangehen im Detail nach und bleibt glaubhaft dicht an dem, was die Mädchen mit ihr in diversen Sitzungen geteilt haben. Der Umfang der Zitate stört zwar manchmal den Lesefluss, aber genau dies zwingt zum Einlassen auf real existierende Mädchen. Auch wenn die Themen und der Alltag der Mädchen deutlich US-amerikanisch geprägt sind, zeigen sie Grundstrukturen vom Mädchen-Sein heute auf, die über den nationalen Bezug hinaus bedeutsam sind. Die sorgsam geführte Erhebung und Auswertung erweist sich als ein sehr gelungenes Beispiel für qualitative Forschung mit Mädchen. Der Dissertation gelingt es, einen fruchtbaren Beitrag zur Diskussion über das Mädchen-Sein heute zu leisten.

Maya Götz
Daniel Hajok / Olaf Selg / Achim Hackenberg (Hrsg.)

\section{Auf Augenhöhe?}

Rezeption von Castingshows und Coachingsendungen

Konstanz: UVK, 2012. - 271 S.

(Alltag, Medien und Kultur; 10)

ISBN 978-3-86764-344-3

Der Sammelband mit dem beziehungsreichen Titel „Auf Augenhöhe?“ ist als Bd. 10 der Reihe „Alltag, Medien, Kultur“ erschienen und versammelt in einem theoretischen und einem empirischen Teil neben der Einleitung von Joachim von Gottberg insgesamt 18 Beiträge in zwei thematischen Hauptteilen. Kern der Publikation bilden Rezeptionsstudien eines zusammenhängenden Forschungsprogramms bzw. von Untersuchungen der AGJM (Arbeitsgemeinschaft Jugendmedien) zu Casting- und Coachingsendungen. Die thematische Ausrichtung hinsichtlich der Stellung, Rolle und Wirkung von sog. Factual Entertainment Formaten, darunter Angeboten des Reality TV, die im letzten Jahrzehnt eine beispiellose Ausbreitung in der fernsehspezifischen Inszenierung von verschiedenen Themen-, Problem- und Alltagbereichen hinweg erfahren haben, ist zwar nicht neu, sie scheint aber für jede aktuelle Formatentwicklung wiederholt behandelt und neu gestellt werden zu müssen. Ein Grund besteht darin, dass Aussagen aus den frühen 2000er Jahren zu Daily Talks oder „Big Brother" angesichts der schnellen Abfolge von Formatentwicklungen, aber auch angesichts immer neu heranwachsender "Mediengenerationen “ mit jeweils unterschiedlichen Medienmenüs, nicht einfach übertragen werden können, sondern beständig überprüft werden müssen. Somit sind es nun also die Coaching- und Castingsendungen wie „Die Super Nanny" oder "DSDS“ und "GNTM“, die in diesem Sammelband vor allem jungendschutzrelevante Fragen auf sich ziehen. Es handelt sich um Fragen danach, welche Vorbildrolle, Wertevermittlung und Identifikation - ein nach wie vor viel zu facettenreicher Begriff für die Rezeptionsforschung - diese Formate leisten.

Eingeleitet wird der Sammelband im ersten, „Perspektiven auf Reality-TV“ genannten Teil mit fünf Beiträgen, die sich genre- und formatbezogenen Fragen widmen und die eine fernsehspezifische Einordnung verfolgen. Den größeren Umfang nehmen im zweiten, „Empirische Befunde" genannten Teil zwölf Berichte zu empirischen Studien zum Einfluss von Casting- und Coachingsendungen auf Kinder und 
Jugendliche ein. Der überwiegende Teil der dort versammelten Beiträge behandelt medienpädagogische Fragestellungen und Herausforderungen. Die drei Herausgeber sind in unterschiedlicher personeller Zusammensetzung an fünf der zwölf empirischen Studien als Autoren beteiligt.

Die Beiträge im ersten Teil behandeln unterschiedliche Problemstellungen zum Themenkomplex „Reality-TV“, von denen sich jedoch nur drei Beiträge im engeren Sinne mit den im Band thematisierten Casting-Shows oder Coaching-Sendungen befassen. Es handelt sich um ein Interview von Joachim von Gottberg mit Ute Biernat zur Position von Grundy Light Entertainment sowie um einen Beitrag von Claudia Mikat zu Aspekten des Jugendmedienschutzes. Eine formatspezifische Einordnung verfolgt der Beitrag von Gräßer und Riffi. Eine über diese drei verschiedenen Textformen hinausweisende Rahmung verfolgt ein Beitrag von Friedrich Krotz zu „Reality-TV“ und dessen gesellschaftlicher Stellung und Bedeutung, sowie ein gemeinsamer Beitrag von Bernhard Pörksen und Wolfgang Krischke zum Wandel der Wahrnehmung von „Vorbildern“ mit dem Titel „Die Gesellschaft der Beachtungsexzesse".

Die zwölf Beiträge zu den empirischen Studien stellen deren Ertrag im Wesentlichen in Kurzform dar. Der Textform nach handelt sich also um Zusammenfassungen von unterschiedlichen Studien zu den genannten Formaten mit zum Teil durchschnittlicher Qualität, was vor allem daran liegt, dass fünf Studien ohne Fazit oder einer zusammenfassenden Diskussion zur Reichweite der Ergebnisse enden. Auf die Sendung DSDS mit Dieter Bohlen und GNTM mit Heidi Klumm entfallen die meisten Beiträge dieses Buchteils. Neben vier Studien der Herausgeber zu den Casting-Shows finden sich Beiträge unter anderem von Tanja Thomas und Miriam Stehling zu GNTM sowie von Margreth Lüneborg und Claudia Töpper zur Skandalisierung in Coaching und Castingshows und von Ulrike Prokop zusammen mit Anna Stach zur Neucodierung von Weiblichkeit in diesen Sendungen. Der Faszination Casting-Shows widmen sich neben den Herausgebern in ihren Beiträgen auch Maya Götz und Johanna Gather in ihrem Artikel, mit dem der empirische Teil eingeleitet wird. Abgeschlossen wird dieser Teil mit einer Studie zur Sendung „Die Super Nanny" von Jan Keilhauer oder Karin Schleider, Gesa Argow und Olaf Selg.

Der Ertrag aller Studien hätte für den am Thema interessierten Leser durch einen diesem Buchteil vorangestellten einleitenden und die
Ergebnisse zusammenfassenden Beitrag deutlich übersichtlicher und leichter aufnehmbar gestaltet werden müssen. Durch das Fehlen einer solchen Zusammenfassung überwiegt eine Disparatheit der empirischen Perspektiven und Zugangsweisen zu den Kernfragen. Dieses Defizit kann man aber nicht diesem Band alleine anlasten, sondern es findet sich in vielen Publikationen und sogar auf Tagungen reproduziert, in denen Studien zu vergleichbaren Themen beziehungslos nebeneinanderstehen.

Auch die Thematik des Buches ist trotz des Formatwechsels von Reality-Shows wie „Big Brother" zu Casting- und Coachingsendungen nicht neu. Reality TV und die Flut der Daily Talkshows galt fast einer Dekade vor den im Band behandelten Formaten die zentrale Aufmerksamkeit, woran der Beitrag von Krotz richtigerweise erinnert und eine Reihe von Motivschichten und Fragestellungen anführt, die seitdem an immer wieder neue Formatinnovationen herangetragen wurden.

Die Innovation in der Forschung bleibt mit diesem Sammelband hingegen aus, wenn an Heranwachsende die immer wieder gleichen Fragen nach Vorbildrolle, Identifikation oder Bedeutung der Realitätsnähe dieser Sendungen gestellt wird. Die Antworten hierauf sollten sich im Vergleich zu früheren Dekaden kaum maßgeblich verändert haben, solange Identität, Werte, Vorbildfunktion isoliert von sozialen Veränderungen thematisiert und nicht die Frage nach den veränderten Bedingungen und Voraussetzungen der mit den Formaten verbundenen Realitäts- und Weltbildkonstruktionen gestellt wird. Die Augenhöhe im Hinblick auf diese Fragen ist von der Forschung also erst herzustellen und wird im Band nur von wenigen Beiträgen wie etwa dem von Thomas und Stehling auf empirischem Gebiet eingelöst. Somit mag der Sammelband für die am Jugendschutz interessierten Kreise einen wichtigen Beleg dafür liefern, dass man die aktuellen Veränderungen in den Blick nimmt und bewertet. Aus wissenschaftlicher, u. a. an Fragen der Medienrezeption und -aneignung interessierter Perspektive vermag der Band wegen der fehlenden integrierenden Betrachtung der Studienergebnisse jedoch nicht abschließend $\mathrm{zu}$ überzeugen.

Udo Göttlich 